\title{
Next Lepton
}

\author{
Yu. A. Baurov \\ Closed Joint Stock Company, Research Institute of Cosmic Physics, 141070, \\ Moscow Region, Korolyov, Pionerskaya, 4, Russia \\ E-mail address: baurov@mail.ru
}

\begin{abstract}
The physics of lepton masses nature is shown on the base of theory of byuons, the theory of "life' of special unobservable discrete objects - byuons from which the surrounding space and the world of elementary particles are formed. An essential distinction of that theory from the modern models in the classical and quantum field theories is that the potentials of physical fields (gravitational, electromagnetic, asf.) gain exactly fixable, measurable values. The theory of byuons predicts the existence of a next lepton $(80,4 \mathrm{GeV}$.) which mass is very same with $\mathrm{W}$-boson mass.
\end{abstract}

Keywords: byuon; leptons; next lepton; W-boson

\section{INTRODUCTION}

It is known that standard model of modern physics has six leptons (e - electron, $v_{\mathrm{e}^{-}}$ electron neutrino; $\mu$ - muon, $v_{\mu}$ - muon neutrino; $\tau$ - lepton, $v_{\tau}-\tau$-neutrino) [1]. In the paper it will predicted that nature has next lepton in the framework of the byuon theory. The theory of byuons is theory of "life' of special unobservable discrete objects - byuons from which the surrounding space and the world of elementary particles form. An essential distinction of the byuon theory from modern models in the classical and quantum theories of field is that the potentials of physical fields (gravitational, electromagnetic, asf.) become, in the said theory exactly fixable, measurable values. Basic axioms and some conclusions of this theory are discussed in [2-7] (see Appendix too).

\section{THEORY OF BYUON AND MASS OF LEPTONS}

What is mass, and why is it different for various elementary particles? Consider character of mass origin for the most simple group of elementary particles, so-called "leptons". In the physics of elementary particles there are six leptons [1]: electron, muon, tau-lepton, and corresponding neutrinos. There are introduced lepton charges for electron, muon, and taulepton. It is known that [1] in the existent physics there is no general interpretation of charges ascribed to particles. But in the theory of byuons [2], we give a rigorous formulation of lepton charge. Namely, at particles identified with leptons, there always exist and never disappear vacuum state (VS) $\mathrm{I}^{+}$for particles and VS I ${ }^{-}$for antiparticles (See Appendix, [2-7]). These VSs close information in themselves during formation of physical space. In Table 1 shown is 
the correspondence between electron, positron, muon, antimuon, (corresponding neutrino antineutrino) and lepton numbers L and associated VSs and psi-functions ( $\psi_{e-}, \psi_{e^{+}}, \psi_{\widetilde{v}_{e}}$, et. al.) - we should not forget that VSs of byuons have a probabilistic character [2].

Table 1. Lepton numbers.

\begin{tabular}{|c|c|c|c|}
\hline$\psi_{e^{-}}\left(I I^{+}, I^{+}\right)$ & $\psi_{e^{+}}(I I, I)$ & $\psi_{v_{e}}\left(I I^{+}, I^{+}\right)$ & $\psi_{\tilde{v}_{e}}(I I, I)$ \\
$L_{e}=+1$ & $L_{e}=-1$ & $L_{e}=+1$ & $L_{e}=-1$ \\
\hline$\psi_{\mu-}\left(I I^{+}, I^{+}\right)$ & $\psi_{\mu^{+}}(I I, I)$ & $\psi_{v_{\mu}}\left(I I^{+}, I^{+}\right)$ & $\psi_{\tilde{v}_{\mu}}(I I, I)$ \\
$L_{\mu}=+1$ & $L_{\mu}=-1$ & $L_{\mu}=+1$ & $L_{\mu}=-1$ \\
\hline
\end{tabular}

Physical nature of lepton mass consists in a residual potential energy of byuons interaction remained after minimization of potential energy of byuon VSs interaction in the one-dimensional space $\mathrm{R}_{1}$ formed by them [2]. Explain that by the example of electron. The expression for electron mass (self-energy) $\mathrm{m}_{\mathrm{e}}$ is

$$
\mathrm{m}_{\mathrm{e}} \mathrm{c}^{2}=\mathrm{m}_{4 \sigma^{*}} \mathrm{c}^{2}\left(\mathrm{ct}^{*} / \mathrm{x}_{\mathrm{o}}\right)
$$

Here the energy $m_{4 b} \cdot c^{2}$ is equal to the self-energy of object $4 b$ (object formed during fourcontact byuon-byuon interaction in VSs $\mathrm{II}^{+} \mathrm{I}^{+} \mathrm{I}^{-} \mathrm{II}^{-}$(to read as " $4 \mathrm{~b}$-object", $\mathrm{mc}^{2}{ }_{4 \mathrm{~b}}=33 \mathrm{eV}$.)) that always finds itself, with probability 1 , in the limits of first period of interaction of byuon VS, which period equals $x_{0} \approx 0,897 * 10^{-17} \mathrm{~cm}$ during electron formation [2].

The quantity (ct*/x $x_{0}$ ) is equal to $\mathrm{N}=15444$, the period in $i$ (index $i$ is a quantum number for byuon ( $\mathrm{ct}^{*} \approx 10^{-13} \mathrm{~cm}$ ), see Appendix). The expression (1) gives an accurate experimental value for the electron mass. Masses of the muon and tau-lepton are similarly found in [2] (see below). But for two last mentioned particles, the objects $4 \mathrm{~b}$ are always in the limits of $2 x_{0}$ and $8 x_{0}$, respectively, with probability 1 . In this connection these particles are prone to decay since the nature tends to the minimum period in $i$ when creating their physical space $x_{0}$.

It should be but noted that the potential energy of byuon VSs interaction, remained after minimization, has, in the case of muon, tau-lepton and next lepton etc., its own value different from those for electron (that is, the own objects $4 b$ ).

In the theory of byuons the above mentioned residual energy for the electron, muon, and tau-lepton at scales $x_{0}, 2 x_{0}$, and $8 x_{0}$, respectively, are brought into line to pairs "neutrinoantineutrino" (that is, to objects $4 \mathrm{~b}$ in our terminology). Leptons are described by VSs $\mathrm{II}^{+}$and $\mathrm{I}^{+}$.

On basic of expression physics (1) we give a values for the lepton masses 
1. $\mathrm{m}_{\mathrm{e}} \mathrm{c}^{2}=\mathrm{m}_{4 \delta_{\mathrm{e}}} \cdot \mathrm{c}^{2} \cdot \frac{\mathrm{ct}}{\mathrm{x}_{0}} \approx 0,51 \mathrm{M \ni B}$

$$
\mathrm{m}_{4 \delta_{\mathrm{e}}} \mathrm{c}^{2}=\frac{\left|\mathrm{A}_{\mathrm{g}}\right|^{2} \cdot \tilde{\mathrm{x}}_{0}^{3}}{2 \mathrm{c}^{0} \cdot \tau_{0}^{2}}
$$

2. $\mathrm{m}_{\mu} \mathrm{c}^{2}=\mathrm{m}_{4 \delta_{\mu}} \cdot \mathrm{c}^{2} \cdot \frac{\mathrm{ct}^{*}}{2 \mathrm{x}_{0}} \approx 105,5 \mathrm{MэB}$

$$
\mathrm{m}_{4 \delta_{\mu}} \cdot \mathrm{c}^{2}=\frac{\left|\mathrm{A}_{\mathrm{g}}\right|}{\mathrm{ct}^{*}} \cdot \frac{\mathrm{A}^{0}}{2 \mathrm{x}_{0}} \cdot \frac{2 \mathrm{x}_{0}}{\mathrm{ct}^{*}} \cdot 2 \mathrm{x}_{0}^{3}
$$

$$
\mathrm{A}^{0}=\frac{2 \sqrt{3} \cdot \mathrm{e}_{0}}{\sqrt{\mathrm{x}_{0} \cdot \mathrm{ct}^{*}}}
$$

3. $\mathrm{m}_{\tau} \mathrm{c}^{2}=\mathrm{m}_{4 \delta_{\tau}} \cdot \mathrm{c}^{2} \cdot \frac{\mathrm{ct}^{*}}{8 \mathrm{x}_{0}} \approx 1785 \mathrm{MэB}$

$$
\begin{gathered}
\mathrm{m}_{4 \delta_{\tau}} \cdot \mathrm{c}^{2}=\frac{\left|\mathrm{A}_{\mathrm{g}}\right|}{c \mathrm{t}^{*}} \cdot \frac{\mathrm{A}_{\tau}^{0}}{2 \mathrm{x}_{0}} \cdot \frac{2 \mathrm{x}_{0}}{c \mathrm{t}^{*}} \cdot 24 \cdot 8 \mathrm{x}_{0}^{3} \\
\mathrm{~A}_{\tau}^{0}=\frac{2 \sqrt{3} \cdot \mathrm{e}_{0}}{\sqrt{2 \mathrm{x}_{0} \cdot \mathrm{ct}^{*}}}
\end{gathered}
$$

4. $\mathrm{m}_{\mathrm{nx}} \mathrm{c}^{2}=\mathrm{m}_{4 \delta_{\mathrm{nx}}} \cdot \mathrm{c}^{2} \cdot \frac{\mathrm{ct}^{*}}{512 \mathrm{x}_{0}} \approx 80,4$ ГэВ

1.

$$
\mathrm{m}_{4 \delta_{\mathrm{nx}}} \cdot \mathrm{c}^{2}=\frac{\left|\mathrm{A}_{\mathrm{g}}\right|}{\mathrm{ct} *} \cdot \frac{\mathrm{A}_{\mathrm{nx}}^{0}}{2 \mathrm{x}_{0}} \cdot \frac{2 \mathrm{x}_{0}}{c \mathrm{t}^{*}} \cdot 1536 \cdot 512 \mathrm{x}_{0}^{3}
$$

$$
\mathrm{A}_{\mathrm{nx}}^{0}=\frac{2 \sqrt{3} \cdot \mathrm{e}_{0}}{\sqrt{4 \mathrm{x}_{0} \cdot \mathrm{ct} \mathrm{t}^{*}}}
$$

In expressions (2) we offered that" 4 b-object" always finds itself, with probability 1 , in phase volumes $\mathrm{x}_{0}^{3}, 2 \mathrm{x}_{0}^{3}, 8 \mathrm{x}_{0}^{3}, 512 \mathrm{x}_{0}^{3}$, ( first period of interaction of byuon VS in $i$, see sequence: $2^{0}, 2^{1},\left(2^{1}\right)^{3},\left(\left(2^{1}\right)^{3}\right)^{3}$ etc. We took into account in (2) the phenomenon of charge 
formation to electroseparating processes described in [2] and the formation an effective "selfaction" potentials for these particles too [2].

\section{DOES NEUTRINO REALLY EXIT?}

As is known [8], neutrino (electron-type one) was discovered by Pauli "on the tip of his pen", on the basis of analysis of conservation laws. Recently, a number of papers [9-17] has evolved which extends considerably our knowledge of neutrino physics.

It was shown by various research teams on the basis of investigation of tritium spectrum end that mass of neutrino is less than zero [9-14].

In Ref. [15], results of precision measurements of tritium spectrum end are presented which suggest that mass squared of the electron-type neutrino $m_{v_{e}}^{2}<0$. This artifact is explained in Ref. [16] with the aid of long range interactions of anomalous neutrinos through introducing a potential, which is equal to the sum of those of Yukawa's type.

In Ref. [17], these unique experimental results are explained by introducing a weakly interacting light scalar boson through which the neutrinos interact in some cloud. It is shown in so doing that the parameters of neutrinos will be cloud density dependent. This work develops the assumption of Ref. [96] that for explaining the experiments, the density of neutrinos $10^{13}$ times greater than that accepted in cosmology is necessary.

In the new physical conception [2,3] of forming the observable three-dimensional physical space $R_{3}$ from a finite set of one-dimensional objects (byuons) in consequence of their dynamics in a one-dimensional space $\mathrm{R}_{1}$, the most simple object, under which the author means the electron-type neutrino, has just the imaginary proper rest energy $\left(m_{v_{e}}^{2} \mathrm{c}^{2}\right)$ in $\mathrm{R}_{1}$ and the imaginary quantum number, mass $m_{v_{e}}^{2}$, in $\mathrm{R}_{3}$. That is why the question in the heading of this article has arisen: "Does the neutrino really exist?"

All experiments proving the existence of neutrino may be divided into two groups in the first of which we are dealing with the circumstantial evidence, starting from the conservation laws, that neutrinos exist [18,19] (among these are also the papers [10-15]), whereas the second group of experiments has pretensions to the direct corroboration of neutrino existence $[20,21]$.

Let us consider the first experiment on detecting neutrino, which was carried out by A. I. Leipunsky [22] and relates to the first group of above mentioned experiments. The idea of his experiment was constructed on comparison of energetic spectra of electrons and recoil nuclei produced during the $\beta$-decay.

A. I. Leipunsky, when investigating the process of $\beta^{+}$-decay in his experiments with the carbon isotope ${ }_{6}^{4} \mathrm{C}$ has validated the inequality $\left(\left|P_{e}\right| \neq\left|P_{r, n}\right|\right.$, where $P_{e}$ is momentum of $\beta$ electron, $P_{r, n}$ is momentum obtained by the recoil nucleus during $\beta$-decay) and thereby, as he believed, proved the existence of neutrino. The Leipunsky's experiment and all those in the first group can be explained on the assumption that the conservation laws of momentum, angular momentum lepton charge etc, are taken over by a large-scale object, the physical space (vacuum medium $[2,3,7]$ ) of the Universe.

An analogy to such a phenomenon is the known Mössbauer effect [22] lying in the fact that the resonant absorption of $\gamma$-radiation by nuclei in conditions of partial overlap of emitted and absorbed $\gamma$-radiation lines rises sharply when the radiation source and absorbent are cooled. 
The essence of the Mössbauer effect is that an oscillator being in the state with minimum energy can, within the framework of quantum model of solid body (the solid body is a set of harmonic oscillators [22]), solely acquire energy but cannot give it up.

An analogous pattern is observed also in the experiment of A. I. Leipunsky as well as in other experiments, for instance, in decay of neutron:

$$
n \rightarrow p+c^{-}+\widetilde{v}_{e}
$$

if assumed that the physical space is a certain periodic oscillating medium taking over the laws of conservation, i.e. one does not need to introduce into the reaction (3) the electron-type antineutrino as it was done by Pauli in order to meet the conservation laws, but in this case the event (3) may not already be considered as a local phenomenon.

Let us show that on the theoretical basis (see Appendix and [2,3]).

Recall that the vacuum states $I^{+}, I^{+}, I, I I$ of the byuons appear randomly and are characterized for the byuon $\Phi(i)=A_{G} x(i)$ by special functions $\Psi_{I I^{+}}^{i}, \Psi_{I^{+}}^{i}, \Psi_{I^{-}}^{k-i}$, and $\Psi_{I I^{-}}^{r-i-2}$, respectively. The product $\Psi_{I I^{+}}^{i+2} \cdot \Psi_{I^{+}}^{i}$ means $[2,3$,$] the probability of existence of vacuum states$ $I I^{+}$and $I^{+}$of the byuon with the index $(i+1)$. The vacuum state $I I^{+}$increases the index $i$ of a byuon, i.e. the value $x(i)$, by one, and the vacuum state $I^{+}$decreases it by one. Normalizing the functions $\Psi_{I^{+}}, \Psi_{I^{+}}, \Psi_{I^{-}}, \Psi_{I^{-}}$is given $[2,3,7]$.

It turns out to be remarkable and surprising that, for the four-contact interaction of byuons ( $4 b$-objects) the equations for the $\Psi$-function have the form of harmonic oscillator [2, 3].

It is shown $[2,3]$ that the object $4 b$ is determined with probability 1 on the characteristic dimension in $R_{3}$ equal to $\sim 10^{-17} \mathrm{~cm}$ (the completion of forming $R_{3}$ ).

It was shown in [2,3] too that the charge and quantum numbers of elementary particles are formed on distances of $10^{-17}-10^{-13} \mathrm{~cm}$, and to the free object $4 b$ there corresponds in $R_{3}$ an uncertainty in coordinate equal to $10^{28} \mathrm{~cm}$ (in accordance with the Heisenberg uncertainty relation)[2,3]. The residual (from minimization process) potential energy in $R_{l}$ is considered in $[2,3]$ as a rest energy $m c^{2}$ in $R_{l}$. The energy of the object $4 b$ equivalent to $m c^{2}$ is equal to $33 \mathrm{eV}$. This object is a boson with spin 1 , there corresponds a pair of electron-type neutrinoantineutrino $\left(v_{e} \Leftrightarrow \widetilde{v}_{e}\right)$ to it. The neutrino is a spinor produced by the interaction of byuons in VS $I I^{+} I^{+}$, but its $m c^{2}$ is imaginary.

The totality of objects $4 b$ over the Universe gives the observed density of matter $\sim 10^{-}$ ${ }^{29} \mathrm{~g} / \mathrm{cm}^{-3}$ (dark matter) and connects all the objects of the Universe into a single information field.

Let us return to reactions of (3)-type.

Thus it is stated that the momentum, energy, spin, and lepton charge attributed to the antineutrino are given up, when the reaction (3) proceeds, to the physical space formed as a result of minimizing the potential energy of byuons in $R_{l}$ and observed by us as an assemblage of the objects $4 b$ which is developing constantly due to the vacuum state $I I^{+}$and creating an oscillating system being at the lowest energy level. Well, but what of the experiments with direct detection of neutrino which particle is observed repeatedly in leading nuclear laboratories in the world, for example, the known experiment of Cowen and Reiness [20,21]:

$$
\widetilde{v}_{e}+p \rightarrow n+e^{+}
$$


An answer is simple. The nuclear reactors near which the reaction (4) is observed due to the reaction (3), create around themselves an unobservable field of objects (with imaginary self-energy) produced during interaction of byuons in the vacuum states $I I \Gamma$ (antineutrino), which objects in turn, when connected with the objects created by byuons in the vacuum states $I^{+} I I^{+}$, give bosons being in the vacuum states $I^{+} I I^{+} I I I$. The set of these bosons forms the physical space, or the space of the elementary particles. The laws of conservation are therewith met to a high accuracy, however not in a local form but in a volume with a characteristic dimension of $\sim 10^{5} \mathrm{~cm}$.

Let us show this.

According to the basic hypothesis (see Appendix and [2,3]), the $4 b$ objects form, as said above, the interior space of an elementary particle along with all its quantum numbers and charges. Therefore such an object always creates, due to its perpetual dynamics in the space $R_{3}$, the minimum momentum for an elementary particle as an integer entity whose interior geometrical space is formed by it.

The momentum of the $4 b$ object corresponding to the minimum momentum for elementary particles, may be represented in a general form $[2,3]$ as

$$
P=\Phi \cdot E_{k_{\text {min }}}^{O} / c
$$

where $\Phi$ is probability of detecting the $4 b$ object in some region of the space $R_{3} ; E_{k_{\min }}^{0} \approx$ $33 \mathrm{eV}$.

If $4 b$ objects are free (i.e. they create not an elementary particle but a space free of elementary particles), then $\Phi=\frac{1}{16} \cdot \frac{\widetilde{x}_{0}^{3}}{4 \pi x_{0}^{2} \widetilde{x}_{0}}$ where $\tilde{x}_{0} \approx 10^{-33} \mathrm{~cm}, x_{0} \approx 10^{-17} \mathrm{~cm}[2,3]$. In this case, if the range of momentum values for an elementary object $\Delta p$ is set equal to $P$, the uncertainty in coordinate in $R_{3}$ for the $4 b$ object will comprise $10^{28} \mathrm{~cm}$.

If an object $4 b$ is not free (i.e. it forms the interior geometry of an electron, as an example), then

$$
\Phi=\frac{1}{16} \cdot \frac{x_{0}^{3}}{4 \pi\left(N x_{0}\right)^{2} x_{0}}
$$

and for a set of $N$ objects $4 b$ forming an electron (for which $m_{e} C^{2}=N E_{k_{\min }}^{0}$ ) we may write

$$
\Delta p=\frac{1}{16} \cdot \frac{x_{0}^{3}}{4 \pi\left(N x_{0}\right)^{2} x_{0}} \cdot \frac{N E_{k_{\text {min }}}^{o}}{c}=\frac{1}{64 \pi} \cdot \frac{E_{k_{\text {min }}}^{o}}{N c}
$$

Then using Eq. (6), we have for $N$ objects $4 b$ an uncertainty in coordinate $\Delta x$ on the order of $10 \mathrm{~cm}$ in $R_{3}$, i.e. an electron, due to wave properties of $N$ objects $4 b$, carries information on its properties not over distances of $10^{-8} \mathrm{~cm}$ (de Broglie wave at the temperature $\sim 300 \mathrm{~K}$ ) as in the case if it were considered as a point-like particle, but at distances of the order of $10 \mathrm{~cm}$.

If one considers not $N$ objects $4 b$ in an electron but only one $4 b$ object (however in an electron, i.e. $\Phi$ is determined by formula (5)), then $\Delta x \sim 10^{5} \mathrm{~cm}$. Thus a lesser quantity of information on the state of interior space characteristics of an electron has a greater spread in coordinate. 
Hence in the range of uncertainty in coordinate $\sim 10^{5} \mathrm{~cm}$ around an electron and presumably a neutron, the processes associated with transmitting energy, momentum etc, to the free space as well as to other objects in accordance with the reaction (3) and (4), can occur. As is seen, the overlap of the processes (3) and (4) is enormous if the reaction (4) is detected even at a distance of hundreds meters from the reactor, which, let us underline it once again, is in perfect analogy to the resonant absorption of $\gamma$-radiation by nuclei and to the Mössbauer's effect as well, however not in coordinates $\Delta E, \Delta t$, but $\Delta P, \Delta x$. One can do a simple estimation of the cross-section of the reaction (4) using our approach if to represent it in the form $\sigma=1 / n \lambda$ where $n$ is concentration of nucleons in a nucleus, and $\lambda$ is the maximum uncertainty interval for the new information field of the nucleon which field makes possible the intersection of the reactions (3) and (4). When putting $n=10^{38} 1 / \mathrm{cm}^{3}$ and $\lambda \sim 10^{5} \mathrm{~cm}$, as said above, we have $\sigma \sim 10^{-43} \mathrm{~cm}^{2}$. As is known, just such a value of $\sigma$ is observed in the experiment with neutrinos from a reactor as well as found on the basis of standard phenomenological theories $[1,22]$.

\section{W-BOSON AND NEXT LEPTON}

In [23] the W-boson mass (MW) was measured using data corresponding to $2.2 \mathrm{fb}-1$ of integrated luminosity collected in p-p collisions with the CDF II detector at the Fermilab Tevatron collider. It was showed that MW $=80387 \pm 12$ stat \pm 15 syst $=80387 \pm 19 \mathrm{MeV} / \mathrm{c}^{2}$. This is the most precise measurement of the W-boson mass today and significantly exceeds the precision of all previous measurements combined. We see MW is equal the mass of next lepton. It is not a fortune because we see a new force of nature acting on $\beta$-decay of ultimate particles [24-31]. These experiments point on existence of quantum medium - physical space oscillation (dark matter [3,7]) which can act on $\beta$-decay of ultimate particles and take over itself the conservation laws of momentum, angular momentum, lepton charge etc..

\section{CONCLUSION}

The mass of the W-boson is an important part of the standard model (SM) of particle physics. Therefore I think SM is not the end of physics development because MW is equal the mass of predicted next lepton.

\section{Reference}

[1] L. B. Okun, Leptons and Quarks, "Nauka", Moscow, in Russian, 1990, 346p.

[2] Yu. A. Baurov, On the structure of physical vacuum and a new interaction in Nature (Theory, Experiment and Applications). Nova Science, NY, 2000, 217p.

[3] Yu. A. Baurov, Global Anisotropy of Physical Space. Experimental and Theoretical Basis, Nova Science, NY, 2004, 166p.

[4] Yu. A. Baurov, Structure of physical space and nature of electromagnetic fiel, in coll. Work. PHOTON: Old problems in light of new ideas, Nova Science, NY, 2000, p. 259-269. 
[5] Yu. A. Baurov, Structure of physical space and new interaction in nature (theory and experiment) in Proceedings of conf. Lorentz group, CPT and Neutrinos, World Scientific, 2000, p. 342-352.

[6] Yu. A. Baurov, Structure of Physical Space and Nature of de Broglie Waves (Theory and Experiment), Journal "Annales de la Fondation Louis de Broglie" "Contemporary Electrodynamics", 2002, 443-461.

[7] Yu. A. Baurov, I. F. Malov, Journal of Modern Physics 1 (2010) 17-32, ArXiv: 0710.3018v1 [physics. gen.-ph], 16 Oct. 2007.

[8] Collected Scientific Papers by Wolfgang Pauli, eds. R. Kroonig, V.F. Weisskopf. Vol. II, Wiley-Interscience, New York, 1964, p.1316.

[9] R. G. H. Robertson, T. J. Bowles, G. J. Stephenson, Jr. Wark, J. F. Wilkerson, D. A. Knapp, Phis. Rev. Lett. 67 (1991) 957.

[10] E. Holzschuh, M. Fritschi, W. Kundig, Phis. Lett. B 287 (1992) 381.

[11] Ch. Weinheimer et. al., Phys. Lett. B 300 (1993) 210.

[12] H. Kawami et. al., Phys. Lett. B 256 (1991) 105.

[13] W. Stoeffl, D. Decman, Phys. Rev. Lett. 75 (1995) 3237.

[14] A. I. Belesev et. al., INR preprint 862/94, Moscow, 1994.

[15] J. Bonn, V. M. Lobashev, A. Swift, presented by J. Bonn at the XVII Conference on Neutrino Physics and Astrophysics, Helsinki, 13-19 June 1996;

[16] R. N. Mohapatra, S. Nussinov, Can Long Range Anomalous Neutrino Interactions Account for the Measured Tritium Beta Decay Spectrum? E.-print, hep-ph/ 9610311.

[17] G. J. Stephenson Jr., T. Goldman, B. H. J. Mekellar, Neutrino Clouds, E.-print, hep-ph/ 9603392.

[18] A. I. Alikhanov, Weak interactions. Recent investigations of $\beta$-decay, Moscow, 1960.

[19] J. Allen, Neutrino, transl. from English, Moscow, 1960 (in Russian).

[20] F. Reiness, C. L. Cowan, Phys. Rev. 92 (1953) 830.

[21] F. Reiness, C. L. Cowan, UFN 662 (1957) 391.

[22] K. N. Mukhin, Experimental Nuclear Physics, book 1, part 1, "Energoatomizdat", 1993, (in Russian).

[23] T. Aaltonen et.al. Precise measurement of the W-boson mass with the CDF II detector, Arxiv:1203.0275 v1 [hep-ex].

[24] Yu. A. Baurov, V. L. Shutov, Prikladnaya Fizika 1 (1995) 40, (in Russian)

[25] Yu. A. Baurov, A. A. Konradov, V. F. Kushniruk, Yu. G., Sobolev, Scientific Report № E7-97-206, (Dubna, 1997), p.354.

[26] Yu. A. Baurov A. A. Konradov, E. A. Kuznetsov et al., Mod. Phys. Lett. A16 (2001) 2089.

[27] Yu. A. Baurov, Yu. G. Sobolev, Yu. V. Ryabov, V. F. Kushniruk., Phys. Atom. Nucl. 70 (2007) 1825. 
[28] Yu. A. Baurov, N. A. Demchuk, A. Yu. Baurov, A. Yu. Baurov (Jr.), V. B. Dunin, V. V. Tihomirov, S. V. Sergeev, Prikladnaya Fizika 5 (2011) 12. (in Russian).

[29] Yu. A. Baurov, V. A. Nikitin, V. B. Dunin, N. A. Demchuk, A. Yu. Baurov, A. Yu. Baurov (Jr.), V. V. Tihomirov, S. V. Sergeev, arXiv:1304.6885 [nucl-ex].

[30] Yu. A. Baurov, American Journal of Modern Physics 2(3) (2013) 177.

[31] Yu. A. Baurov, Bull. Russ. Acad. Sci. Phys. 76 (2012) 1201.

( Received 29 September 2013; accepted 03 October 2013 )

\section{Appendix}

Any theory begins with axioms, that is, with basic postulates accepted without proof.

Thus, let us assume that there are no space, no time, no world of elementary particles from which all surrounding us physical bodies consist, but there is a object, a byuon $\Phi(i)$, being unobservable in itself and characterized by discrete states (i.e. numbered by the series of natural numbers) having inherent "vectorial" property. The expression for $\Phi(i)$ is

$$
\boldsymbol{\Phi}(i)=\left\{\begin{array}{c}
{\left[\mathbf{A}_{g} x(i)\right],} \\
-\sqrt{-1}\left[\mathbf{A}_{g} x(i)\right]
\end{array}\right.
$$

where $x(i)$ is "length" of the byuon, a real (positive or negative) value depending on the index $i=$ $0,1,2, \ldots \mathrm{k}, \ldots$ Index $\mathrm{i}$ is a quantum number for $\boldsymbol{\Phi}(\mathrm{i})^{1}$. The value $\mathrm{A}_{\mathrm{g}}$ is some inner potential equal, in magnitude, to the cosmological vector-potential $\mathbf{A}_{\mathrm{g}}$, a novel fundamental vectorial constant introduced by the author $[2,3]$. It is not an ordinary potential of any field theory since we have yet no notions of field and its potential. We have to do with a special property of the byuon that we call therefore the inner byuon potential. The dimensionality of $x(i)$ is that of distance, so we will measure $x(i)$ in centimeters $(\mathrm{cm})$, and $\mathbf{A}_{\mathrm{g}}$ in Gauss centimeter $\left(\mathrm{A}_{\mathrm{g}} \approx 1.95 \cdot 10^{11} \mathrm{Gs} \cdot \mathrm{cm}\right)$. That is, our fundamental object is dimensional. That was presumed by many authors which searched for so called "universum" (that thing from which all the other things are built up), since the real world cannot be constructed from the dimensionless 0 and 1 . But the question of dimensionality of that object remained an enigma. Our object, the byuon, has dimensionality of Gs $\times \mathrm{cm}^{2}$, as also have (in CGSE-system) such physical objects as electric charge, magnetic flux, and Dirak's monopole -a hypothetic object not yet detected in experiments.

The entire set of states $\Phi(i)$ can form a one-dimensional space $\mathrm{R}_{1}$ relative to index $i$. In $\mathrm{R}_{1}$, the distance between byuon states (the metrix) is defined as the difference of their "lengths", i.e. between quantities $x(i)$. Such a metrix is known as Archimedean metrix.

Explain the above said. If, for example, the object $\Phi(i)$ has, in one of its states, an enormous magnitude of $x(i)$ equal to $10^{28} \mathrm{~cm}$, and in its another state, $x(i+k)$ is equal to that same enormous

\footnotetext{
${ }^{1}$ It should be explained that the vector $\Phi(i)$ is not an ordinary vector in some space but an object with "inner" vectorial properties that are manifesting themselves when the value $x(i)$ changes in the process of physical space formation.
} 
magnitude of $10^{28} \mathrm{~cm}$ plus $1 \mathrm{~cm}$, then the distance between those states in the one-dimensional space $\mathrm{R}_{1}$ will be an insignificant $1 \mathrm{~cm}$.

Further, the notion of time is introduced. In accordance with our conception, by the discrete time of byuon is meant a sequence of events corresponding to discrete changes in index $i$ of 1 up or down, which means a transition from one state to another. In connection with discrete time, a time quantum $\tau_{0}$ and space quantum $\tilde{x}_{0}$ are introduced in the one-dimensional discrete space $\mathrm{R}_{1}$ formed by states of byuon ( $\tau \approx 0.9 \cdot 10^{-43} \mathrm{~s}, \widetilde{x}_{0} \approx 2.8 \cdot 10^{-33} \mathrm{~cm}$ ). As $\mathrm{R}_{1}$ is discrete, one way to parametrize (represent) $x(i)$ is $x(i) \approx \widetilde{x}_{0} \cdot \mathrm{I}$ or $x(i) \approx-\widetilde{x}_{0} \cdot i$. As is seen, the magnitude of $x(i)$ can be both positive and negative. Similar parameterization for time $t=(\tau \cdot j)$ gives only positive magnitudes of $t$ (here $t$ is an always increasing index taking only integer positive values and the number 0 ). Note that in the existing physics of elementary particles, a notion of negative time is used to introduce antiparticles. This is not necessary in the present model.

Thus the byuon is characterized by indexes $i$ and $j(i \leq j)$. We will consider only the case of $i=j$.

Statics. In the set $\{\boldsymbol{\Phi}(i)\}$, no static states with time $\mathrm{t}>\tau_{0}$ are meant. That is, the byuon is always in state of continuous changing.

Kinematics. A free byuon state (that is, not interacting with another state) can correspond only to one of four so called vacuum states $\mathrm{II}^{+}, \mathrm{I}^{+}, \mathrm{I}^{-}, \mathrm{II}^{-}$depending on whether the vector $\Phi(i)$ is real or imaginary, its length $\mathrm{X}(i)$ positive or negative, decreases or increases in modulus.

Introduce the following definitions:

1. The free byuon is in vacuum state $\mathrm{II}^{+}$if its positive length discretely increases of a quantum of distance $\widetilde{x}_{0}$ in a quantum of time $\tau_{0}$. At that the speed of byuon propagation (increasing in length) $c=\frac{\widetilde{x}_{0}-0}{\tau_{0}}=c_{0}\left(\mathrm{c}_{0}\right.$ is speed of light $)$.

2. The free byuon is in VS I ${ }^{+}$if its lenght discretely decreases of a quantum of distance $\widetilde{x}_{0}$ in a quantum of time $\tau_{0}$. Inthiscase $c=\frac{0-\widetilde{x}_{0}}{\tau_{0}}=-c_{0}$.

3. The free byuon is in $\mathrm{VS} \mathrm{II}^{-}$if the modulus of its negative length discretely increases of $\widetilde{x}_{0}$ in a time $\tau_{\mathrm{o}}$. Therewith $c=\frac{-\widetilde{x}_{0}-0}{\tau_{0}}=-c_{0}$.

4. The free byuon is in VS I- if the modulus of its negative length discretely decreases of $\widetilde{x}_{0}$ in a time $\tau_{0}$. In this case $c=\frac{0-\left(-\widetilde{x}_{0}\right)}{\tau_{0}}=c_{0}$.

Location of byuon in one or another VS is of probabilistic character and described by four $\psi$ functions with indexes corresponding both to VSs and magnitudes of quantum number $i: \Psi_{I I^{+}}^{i+2}, \Psi_{I I^{-}}^{k-i}$, determining the processes of byuon length magnitude origin and increase at positive and negative $X(i)$, respectively; $\Psi_{I^{+}}^{i}, \Psi_{I^{-}}^{k-i-2}$, determining the processes of byuon length magnitude cancellation and decrease at positive and negative $X(i)$, respectively.

Assume that for the byuons with the length greater than $\tilde{x}_{0}$, only contact interactions are realized, by which we will mean existence of at least two byuons at a quantum of space $R_{l}$ (onedimensional space).

Hypothesis 1. Assume the observable three-dimensional space $R_{3}$ to appear as a result of minimization of the potential energy (E(i)) of byuon interaction in the one-dimensional space $R_{1}$ formed by them. More precisely, the space $R_{3}$ is fixed by us as the result of this byuon dynamics. In the space $R_{3}$ therewith the dynamic processes for objects with the residual positive potential energy of byuon interactions originate, and in consequence, the wave properties of elementary particles arise. 
Note that the known fundamental constant, light speed, appears in the byuon theory as a result of byuon changes. In what follows the reader will know that, when having specified only three constants: modulus of $\mathbf{A}_{\mathrm{g}}, \tau_{0}$, and $\widetilde{x}_{0}$, we obtain values of all other fundamental constants and basic properties of the entire world as well. (see Table) $[2,3]$.

Table. Basic results of byuon theory.

\begin{tabular}{|c|c|}
\hline Given & Obtained \\
\hline \multirow{5}{*}{$\begin{array}{l}\text { 1. The quantum of space } \\
\tilde{x}_{0} \approx 2.8 \cdot 10^{-33} \mathrm{~cm} . \\
\text { 2. The quantum of time } \\
\tau \approx 0.9 \cdot 10^{-43} \mathrm{~s} . \\
\text { 3. The module of } \\
\text { cosmological vector- } \\
\text { potential } \mathbf{A}_{\mathrm{g} .} \\
\mathrm{A}_{\mathrm{g}} \approx 1.95 \cdot 10^{11} \mathrm{Gs} \cdot \mathrm{cm}\end{array}$} & $\begin{array}{l}\text { a. The fundamental scales: } x_{0}=\mathrm{k} \widetilde{x}_{0} \approx 10^{-17} \mathrm{~cm} ; \\
\mathrm{ct}^{*}=k \mathrm{~N} \widetilde{x}_{0} \approx 10^{-13} \mathrm{~cm} ; \mathrm{L}=\mathrm{kNP} \widetilde{x}_{0} \approx 10^{28} \mathrm{~cm} ; \\
\mathrm{k}, \mathrm{N}, \mathrm{P}-\text { calculated periods of interaction of byuons. }\end{array}$ \\
\hline & $\begin{array}{l}\text { b. The fundamental constants: speed of light } \mathrm{c}_{0}=\widetilde{x}_{0} / \tau \text {, the } \\
\text { elementary electric charge } \mathrm{e}_{\mathrm{o}}^{2}=(1 /(4 \sqrt{3})) \mathrm{A}_{\mathrm{g}}^{2} \mathrm{x}_{\mathrm{o}}^{2}\left(\mathrm{x}_{\mathrm{o}} / \mathrm{ct}\right)^{3 / 2} \\
\text { Planck's constant } \mathrm{h}=\left(\left(\left[\mathrm{A}_{\mathrm{g}} \mathrm{x}_{\mathrm{o}}\right]_{\mathrm{II}}^{+}\left[\mathrm{A}_{\mathrm{g}} \mathrm{x}_{\mathrm{o}}\right]_{\mathrm{I}}^{-}\right) / \mathrm{c}_{\mathrm{o}}\right) \mathrm{x}_{\mathrm{o}} / \mathrm{ct}^{*}\end{array}$ \\
\hline & $\begin{array}{l}\text { c. The constants of all interactions, for example, vector constant of } \\
\text { weak interaction } \mathrm{C}_{\mathrm{v}}=\mathrm{e}_{\mathrm{o}} \mathrm{A}_{\mathrm{g}} 2 \mathrm{x}_{\mathrm{o}}{ }^{3} \text {. }\end{array}$ \\
\hline & $\begin{array}{l}\text { d. Mass spectra of leptons (electron, muon, tau-lepton asf.), main } \\
\text { barions, and mesons, for example, the mass of electron: } \mathrm{m}_{\mathrm{e}} \mathrm{c}^{2} \\
=\mathrm{m}_{46} \mathrm{c}^{2}\left(\mathrm{ct}^{*} / \mathrm{x}_{\mathrm{o}}\right) \text {; the minimum value of that residual potential } \\
\text { energy: } \\
\qquad E_{k_{\min }}^{0}=\left(A_{g}^{2} \widetilde{x}_{0}^{2}\right) /\left(2 \widetilde{x}_{0}\right)=\mathrm{m}_{46} \mathrm{c}^{2}=33 \mathrm{eV} .\end{array}$ \\
\hline & $\begin{array}{l}\text { e. The density of matter in the Universe } \sim 10^{-29} \mathrm{~g} / \mathrm{cm}^{3} \text { (physics of } \\
\text { dark matter; dark energy: physics on the base of new force, } \\
\text { values galactic and intergalactic magnetic fields and asf. }\end{array}$ \\
\hline
\end{tabular}

